DRAFT VERSION OCTOBER 30, 2018

Preprint typeset using LTEX style emulateapj v. 08/22/09

\title{
PROPER MOTIONS OF THERMAL RADIO SOURCES NEAR HH 7-11 IN THE NGC 1333 STAR FORMING REGION
}

\author{
Carlos Carrasco-González ${ }^{1}$, Guillem Anglada ${ }^{1}$, Luis F. RodrígueZ ${ }^{2}$, José M. Torrelles ${ }^{3}$, MaYra Osorio ${ }^{1}$ \\ (Received 2008 June 4; Accepted 2008 August 30) \\ Draft version October 30, 2018
}

\begin{abstract}
Star forming regions are expected to show linear proper motions due to the relative motion of the Sun with respect to the region. These proper motions appear superposed to the proper motions expected in features associated with mass ejection from the young stellar objects embedded in them. Therefore, it is necessary to have a good knowledge of the proper motions of the region as a whole in order to correctly interpret the motions associated with mass ejection. In this paper we present the first direct measurement of proper motions of the NGC 1333 star forming region. This region harbors one of the most studied Herbig-Haro systems, HH 7-11, whose exciting source remains unclear. Using VLA A configuration data at $3.6 \mathrm{~cm}$ taken over 10 years, we have been able to measure the absolute proper motions of four thermal sources embedded in NGC 1333. From our results we have derived the mean proper motions of the NGC 1333 star forming region to be $\mu_{\alpha} \cos \delta=9$

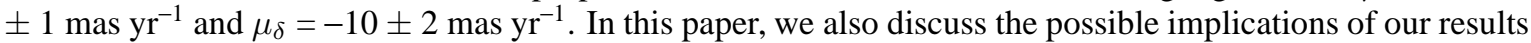
in the identification of the outflow exciting sources.

Subject headings: astrometry — ISM: individual (NGC 1333) — ISM: jets and outflows — radio continuum: ISM — stars: formation — stars: individual (SVS 13)
\end{abstract}

\section{INTRODUCTION}

The stars in nearby regions of star formation are expected to show linear proper motions in the order of milliarcseconds (mas) per year. These proper motions are the result of the relative motion between the Sun and the stars studied, and can be obtained, to first order, by comparing the observed position of the star with respect to the reference frame of the remote quasars at different epochs.

In the last few years, Very Large Array (VLA) observations taken with time baselines of up to two decades have been used to measure these proper motions for regions like Taurus (Loinard et al. 2003), L1527 (Loinard et al. 2002), L1551 (Rodríguez et al. 2003), Ophiuchus (Curiel et al. 2003), and Orion (Gómez et al. 2005).

Even when the VLA observations lack sufficient angular resolution to detect more subtle motions (i.e. geometric parallax), they can provide not only the average proper motion of the region but also reveal the presence of stars moving with peculiar velocities with respect to the region, such as those found in the Orion BN/KL region (Rodríguez et al. 2005, Gómez et al. 2005), that suggest a runaway nature for some of the sources. Much more accurate astrometry can be achieved using Very Long Baseline Interferometry (VLBI) techniques (e.g. Loinard et al. 2007), but these observations are restricted to very compact and bright non thermal radio stars and cannot be applied to thermal sources, where the emission is relatively extended and its brightness temperature is not expected to exceed $10^{4} \mathrm{~K}$, far below the sensitivity of present day VLBI.

The NGC 1333 star forming region, located at a distance of $235 \pm 18$ pc (Cernis 1990; Hirota et al. 2008) in the Perseus complex, harbors the classical bright Herbig-Haro $(\mathrm{HH})$ sys-

\footnotetext{
${ }^{1}$ Instituto Astrofísica Andalucía, CSIC, Camino Bajo de Huétor 50, E-18008 Granada, Spain; charly@iaa.es, guillem@iaa.es, osorio@iaa.es

${ }^{2}$ Centro de Radioastronomía y Astrofísica UNAM, Apartado Postal 3-72 (Xangari), 58089 Morelia, Michoacán, México; 1.rodriguez@astrosmo.unam.mx

${ }^{3}$ Instituto de Ciencias del Espacio (CSIC)-IEEC, Facultat de Física, Universitat de Barcelona, C/ Martí i Franquès, 1, E-08028 Barcelona, Spain; torrelles@ieec.fcr.es
}

tem HH 7-11, first reported by Herbig (1974) and by Strom et al. (1974). The optically visible star SVS 13, discovered as a near-IR source (Strom et al. 1976), is roughly aligned with the chain of $\mathrm{HH}$ objects and was proposed as the powering source of this $\mathrm{HH}$ system. This association was questioned by Rodríguez et al. (1997), who discovered a cm radio source (VLA 3) located $6^{\prime \prime}$ to the SW of SVS 13, and argued that this new object is a better candidate to drive the HH outflow. SVS 13 is also associated with $\mathrm{cm}$ emission (source VLA 4 of Rodríguez et al. 1997) and mm emission (Looney et al. 2000). Bachiller et al. (2000), through interferometric observations, found that SVS 13 is associated with an "extremely" high velocity molecular outflow, although "standard" velocity gas was found in the vicinity of both SVS 13 and VLA 3. Subarcsecond VLA observations at $3.6 \mathrm{~cm}$ and $7 \mathrm{~mm}$ by Anglada et al. $(2000,2004)$ revealed that the radio source associated with SVS 13 is actually a close binary with two components, VLA 4A and 4B, separated by $0 . / 3$ ( 65 AU). Interestingly, a detailed analysis of the positions and spectral energy distribution in the $\mathrm{cm}-\mathrm{mm}$ range suggests that the two sources have very different properties: VLA 4B appears to be associated with the observed strong $\mathrm{mm}$ emission, probably arising from a circumstellar dust disk, while VLA 4A is the counterpart of the visible star SVS 13 with dust emission absent or much less significant in this component.

Since high velocity water masers are indicators of outflow activity and can be observed with high angular resolution, Rodríguez et al. (2002) analyzed archive VLA data of the water masers associated with SVS 13 in order to investigate which of the two stars of the binary system was the most likely candidate to drive the outflow. It was found that the water masers appear segregated in two groups, according to their position and LSR velocity. A group with the LSR velocity similar to that of the ambient cloud is associated with VLA 4A, and a blueshifted velocity group is associated with VLA 4B. This result was interpreted as favoring VLA $4 \mathrm{~B}$ as the outflow driving source. However, the study of Rodríguez et al. (2002) was restricted to the line-of-sight component of the velocity. Recently, Hirota et al. (2008) presented VLBI astrometric 
TABLE 1

OBSERVATIONS PARAMETERS

\begin{tabular}{|c|c|c|c|c|c|c|}
\hline \multirow[b]{3}{*}{ Epoch } & \multirow[b]{3}{*}{$\begin{array}{c}\text { Observation } \\
\text { Date }\end{array}$} & \multicolumn{3}{|c|}{$\overline{\text { Bootstrapped }}$} & \multirow[b]{2}{*}{ Beam $^{b}$} & \multirow[b]{3}{*}{$\begin{array}{l}\text { rms Noise }^{\mathrm{b}} \\
\left(\mu \mathrm{Jy} \text { beam }^{-1}\right)\end{array}$} \\
\hline & & VLA & Flux Density of & Synthesized l & & \\
\hline & & $\begin{array}{l}\text { Project } \\
\text { Code }\end{array}$ & $\begin{array}{c}\text { Phase Calibrator }^{\mathrm{a}} \\
(\mathrm{Jy})\end{array}$ & $\begin{array}{l}\text { HPBW } \\
(\operatorname{arcsec})\end{array}$ & $\begin{array}{l}\text { P.A. } \\
\text { (deg) }\end{array}$ & \\
\hline 1989.1 & 89-Jan-14 & AR202 & $1.343 \pm 0.003$ & $0.29 \times 0.24$ & 62 & 28 \\
\hline 1994.3 & 94-Apr-23 & AR277 & $1.686 \pm 0.006$ & $0.28 \times 0.20$ & -68 & 27 \\
\hline 1996.9 & 96-Dec-22 & AR277 & $1.305 \pm 0.009$ & $0.35 \times 0.30$ & -88 & 24 \\
\hline 1998.2 & 98-Mar-27 & AA218 & $1.476 \pm 0.009$ & $0.26 \times 0.24$ & -5 & 18 \\
\hline 1998.4 & 98-Мау-26 & AA218 & $1.610 \pm 0.010$ & $0.26 \times 0.24$ & -7 & 18 \\
\hline 1999.5 & 99-Jul-03 & AA239 & $1.681 \pm 0.008$ & $0.29 \times 0.25$ & 39 & 17 \\
\hline
\end{tabular}

${ }^{a}$ The phase calibrator used in all the epochs was $0333+321$.

${ }^{\mathrm{b}}$ From naturally weighted maps.

TABLE 2

POSITIONS OF THE SOURCES

\begin{tabular}{|c|c|c|c|c|}
\hline \multirow[b]{2}{*}{ Epoch } & \multicolumn{2}{|c|}{ VLA 2} & \multicolumn{2}{|c|}{ VLA 3} \\
\hline & $\alpha(\mathrm{J} 2000)$ & $\delta(\mathrm{J} 2000)$ & $\alpha(\mathrm{J} 2000)$ & $\delta(\mathrm{J} 2000)$ \\
\hline 1989.1 & $032901.9535 \pm 0.0003$ & $311538.307 \pm 0.005$ & $032903.369 \pm 0.002$ & $311601.89 \pm 0.02$ \\
\hline 1994.3 & $01.9586 \pm 0.0004$ & $38.221 \pm 0.004$ & $03.375 \pm 0.002$ & $01.82 \pm 0.02$ \\
\hline 1996.9 & $01.9605 \pm 0.0004$ & $38.285 \pm 0.008$ & $03.376 \pm 0.001$ & $01.75 \pm 0.02$ \\
\hline 1998.2 & $01.9606 \pm 0.0002$ & $38.188 \pm 0.005$ & $03.376 \pm 0.001$ & $01.72 \pm 0.02$ \\
\hline 1998.4 & $01.9604 \pm 0.0002$ & $38.211 \pm 0.006$ & $03.373 \pm 0.002$ & $01.78 \pm 0.02$ \\
\hline \multirow[t]{2}{*}{1999.5} & $01.9622 \pm 0.0002$ & $38.183 \pm 0.003$ & $03.373 \pm 0.001$ & $01.78 \pm 0.01$ \\
\hline & \multicolumn{2}{|c|}{ VLA 4A } & \multicolumn{2}{|c|}{ VLA 4B } \\
\hline Epoch & $\alpha(\mathrm{J} 2000)$ & $\delta(\mathrm{J} 2000)$ & $\alpha(\mathrm{J} 2000)$ & $\delta(\mathrm{J} 2000)$ \\
\hline 1989.1 & $032903.730 \pm 0.002$ & $311604.02 \pm 0.02$ & $032903.751 \pm 0.002$ & $311604.08 \pm 0.02$ \\
\hline 1994.3 & $03.730 \pm 0.002$ & $03.95 \pm 0.02$ & $03.758 \pm 0.004$ & $04.01 \pm 0.02$ \\
\hline 1996.9 & $03.731 \pm 0.002$ & $03.94 \pm 0.02$ & $03.757 \pm 0.002$ & $04.01 \pm 0.02$ \\
\hline 1998.2 & $\ldots \mathrm{b}$ & $\ldots \mathrm{b}$ & b & $\ldots \mathrm{b}$ \\
\hline 1998.4 & $03.735 \pm 0.002$ & $04.00 \pm 0.03$ & $03.760 \pm 0.002$ & $03.99 \pm 0.02$ \\
\hline 1999.5 & $03.734 \pm 0.002$ & $03.96 \pm 0.02$ & $03.757 \pm 0.001$ & $03.91 \pm 0.02$ \\
\hline
\end{tabular}

${ }^{a}$ Positions derived from Gaussian ellipsoid fits in the naturally weighted maps. Units of right ascension are hours, minutes and seconds, and units of declination are degrees, arcminutes, and arcseconds.

$\mathrm{b}$ The components of the binary system VLA 4 could not be resolved in this epoch.

observations with VERA and derived absolute positions and proper motions of the order of 15-20 mas $\mathrm{yr}^{-1}$ (corresponding to velocities of $16-22 \mathrm{~km} \mathrm{~s}^{-1}$ ) of the water masers associated with SVS 13. These observations confirm the positional association with VLA 4A of the masers with an LSR velocity close to that of the ambient cloud. However, the proper motions (velocity and direction) relative to the sources could not be firmly established because of a poor knowledge of the absolute proper motions of the region. In fact, the optical data of Herbig \& Jones (1983) suggest that the stars projected upon heavy obscuration (presumably associated with the NGC 1333 cloud) have an average proper motion of $\sim 10$ mas $\mathrm{yr}^{-1}$ relative to the stars near the edge of the obscuration (presumably background stars). This suggests that the absolute proper motion of the region could have a significant contribution to the proper motions of the masers observed by Hirota et al. (2008).

In this paper we present an absolute astrometry analysis and proper motion calculations over 10 years of four thermal radio sources in the NGC 1333 region. There are no reports of absolute proper motions for embedded young stars in this region and our study provides the first determination of these motions.
Data at $3.6 \mathrm{~cm}$ continuum were taken using the VLA of the National Radio Astronomy Observatory (NRAO) ${ }^{4}$. The data consist of six epochs of observations, ranging from 1989 January 14 to 1999 July 3, obtained using the A configuration of the VLA, which provides an angular resolution of $\sim 0$.' 3 . A requirement to obtain reliable astrometry is that the same phase calibrator has been used for most or preferably all observations. This requirement is accomplished in our case since all observations were made using $0333+321$ as phase calibrator. Data editing and calibration were carried out using the Astronomical Image Processing System (AIPS) package of NRAO, following the standard VLA procedures. The observation dates, the bootstrapped flux densities of $0333+321$, as well as the parameters of the synthesized beams, and the rms noise of the naturally weighted maps are given in Table 1 Since the nominal position of the phase calibrator used in the first epoch was slightly different from that used in the other five epochs (the positions of the phase calibrators are periodically refined at the VLA), in order to obtain more accurate astrometry, we corrected its position using the task CLCOR of AIPS before calibration of the first epoch. Positions of the four thermal sources (VLA 2, VLA 3, VLA 4A, and VLA 4B; see Rodríguez et al. 1997 and Anglada et al. 2000 for the nomenclature of the sources) were derived in each epoch by

\footnotetext{
4 The NRAO is a facility of the National Science Foundation operated under cooperative agreement by Associated Universities, Inc.
} 

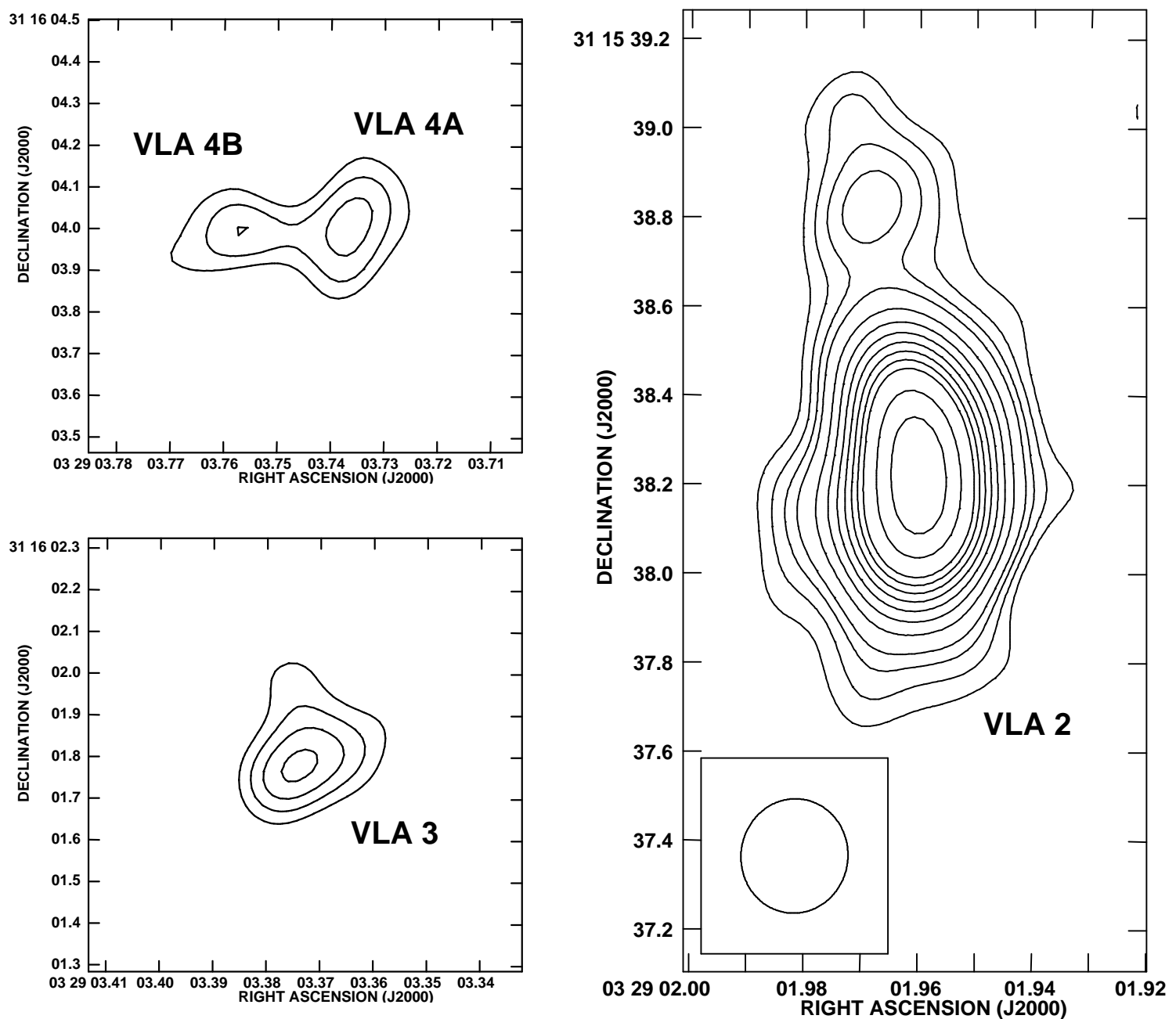

FIG. 1.- Naturally weighted VLA $3.6 \mathrm{~cm}$ continuum map of the thermal sources VLA 2, VLA 3, VLA 4A and VLA 4B at the 1998.4 epoch. Contours are -3, $3,4,5,6,8,10,12,14,16,18,20,25$ and 30 times the rms of the map, $18 \mu \mathrm{Jy} \mathrm{beam}^{-1}$. The synthesized beam, shown in the right hand panel, is 0 !' $^{\prime} 26 \times 0{ }^{\prime \prime} 24$; P.A. $=-7^{\circ}$. The three panels are plotted at the same scale.

Gaussian ellipsoid fits in the naturally weighted maps.

\section{RESULTS AND DISCUSSION}

In Figure 1 we show the 1998.4 maps of the sources VLA 2, VLA 3, VLA 4A, and VLA 4B. The measured absolute positions of each source at each epoch are given in Table 2. In Figure 2 we present position vs time diagrams for each source, and the proper motions, $\mu_{\alpha} \cos \delta$ and $\mu_{\delta}$, obtained by linear least squares fits of these diagrams for each source are given in Table 3. All the sources show proper motions roughly to the SE with P.A. in the range from $\sim 120^{\circ}$ to $\sim 160^{\circ}$ and veloci-

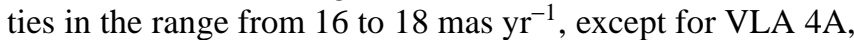
whose velocity is about one half the velocity obtained for the other sources. This possible velocity difference of 8-10 $\mathrm{km} \mathrm{s}^{-1}$ between VLA 4A and the ambient cloud may account for the yet unexplained large increase in the optical and nearIR brightness of VLA 4A that took place in 1988-1990 (Eislöffel et al. 1991). The source may have simply displaced from a very obscured region to a position where it became more easily detectable. However, because of the signal-tonoise limitation of the data plus the errors produced by the intrinsic differences in the morphologies, orientations and flux densities of the sources (see Fig. 1), we cannot conclude that the differences between the values obtained for each source indicate statistically significant differences in the motions of the objects.

As a representative value of the proper motion of the NGC 1333 region as a whole we adopt the weighted average of the proper motion of the four sources, $\mu_{\alpha} \cos \delta=9 \pm 1$

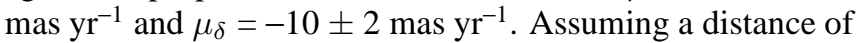
235 pc for NGC 1333, these values imply a velocity of $16 \pm 2$ $\mathrm{km} \mathrm{s}^{-1}$ along a P.A. of $140^{\circ} \pm 10^{\circ}$. This is the first measurement of the proper motion directly made on embedded objects that belong to the NGC 1333 star forming region. Therefore, we believe that our value is, up to date, the most representative of the proper motion of NGC 1333.

The proper motion of the NGC 1333 region obtained from our data can be compared with the value expected due to the effect of the differential galactic rotation and the motion of the Sun with respect to the LSR. Assuming the solar motion with respect to the LSR based on the Hipparcos satellite, $\left(\mathrm{U}_{0}, \mathrm{~V}_{0}, \mathrm{~W}_{0}\right)=(10.00,5.25,7.17) \mathrm{km} \mathrm{s}^{-1}$ (Dehnen \& Binney 1998), the model of Brand \& Blitz (1993) for the galactic rotation, and a distance of 235 pc for NGC 1333, we would ex-

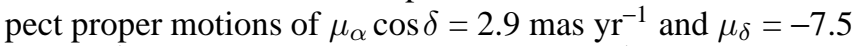
mas $\mathrm{yr}^{-1}$, that imply a velocity of $\sim 9 \mathrm{~km} \mathrm{~s}^{-1}$ along a P.A. of $\sim 160^{\circ}$. This velocity is $\sim 7 \mathrm{~km} \mathrm{~s}^{-1}$ smaller than the value ob- 

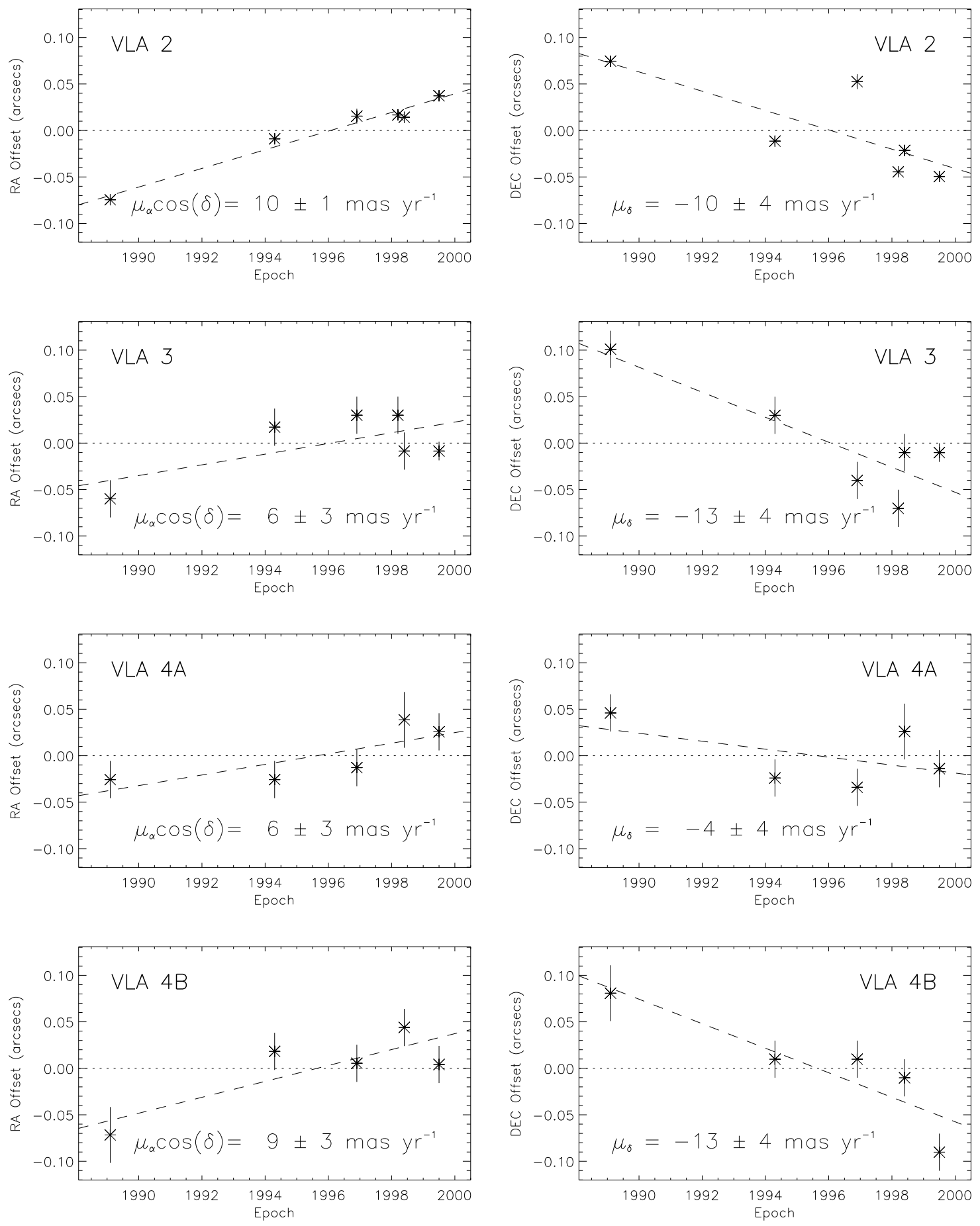

FIG. 2.- Position vs time diagrams of the sources VLA 2, VLA 3, VLA 4A, and VLA 4B. The positions of each source are represented in the diagrams as right ascension and declination offsets (in arcsecs) relative to the mean of the positions given in Table 2 Error bars are obtained from Gaussian ellipsoid fits in the naturally weighted maps. The actual errors could be greater because the morphology of the sources is not exactly Gaussian (see text for details). The dashed line in each panel is a linear least squares fit to the data. The values of $\mu_{\alpha} \cos \delta$ and $\mu_{\delta}$ obtained from these fits are also labeled in each panel. 
TABLE 3

PROPER MOTIONS OF THE SOURCES

\begin{tabular}{cccccc}
\hline \hline Source & $\begin{array}{c}\mu_{\alpha} \cos \delta \\
\left(\mathrm{mas} \mathrm{yr}^{-1}\right)\end{array}$ & $\begin{array}{c}\mu_{\delta} \\
\left(\mathrm{mas} \mathrm{yr}^{-1}\right)\end{array}$ & $\begin{array}{c}\mu \\
\left(\mathrm{mas} \mathrm{yr}^{-1}\right)\end{array}$ & $\begin{array}{c}\mathrm{v} \\
\left(\mathrm{km} \mathrm{s}^{-1}\right)\end{array}$ & $\begin{array}{c}\text { P.A. } \\
(\mathrm{deg})\end{array}$ \\
\hline VLA 2 & $10 \pm 1$ & $-10 \pm 4$ & $14 \pm 3$ & $16 \pm 3$ & $140 \pm 10$ \\
VLA 3 & $6 \pm 3$ & $-13 \pm 4$ & $14 \pm 4$ & $16 \pm 4$ & $160 \pm 10$ \\
VLA 4A & $6 \pm 3$ & $-4 \pm 4$ & $7 \pm 3$ & $8 \pm 3$ & $120 \pm 30$ \\
VLA 4B & $9 \pm 3$ & $-13 \pm 4$ & $16 \pm 4$ & $18 \pm 4$ & $150 \pm 10$ \\
Weighted Mean $^{\mathrm{a}}$ & $9 \pm 1$ & $-10 \pm 2$ & $14 \pm 2$ & $16 \pm 2$ & $140 \pm 10$ \\
\hline
\end{tabular}

a The values of the total proper motion, velocity in the plane of the sky, and P.A. are derived from the mean values of $\mu_{\alpha} \cos \delta$ and $\mu_{\delta}$. 
tained from our data. Since the difference between the proper motion predicted by the model and our measurements is of the order of the one-dimensional rms velocity dispersion observed between molecular clouds $\left(7.8 \mathrm{~km} \mathrm{~s}^{-1}\right.$; Stark \& Brand 1989), we attribute this difference to the peculiar motion of the NGC 1333 cloud. We have looked for optical stars associated with NGC 1333 with proper motions measured by Hipparcos. The only nearby star we find with these characteristics is $\mathrm{BD}+30^{\circ} 549$, a B8:p type star that illuminates part of the reflection nebula NGC 1333 (e.g. Rodríguez et al. 1990). For this star, Hipparcos reports proper motions of $\mu_{\alpha} \cos \delta=$ $6.31 \pm 2.35$ mas yr$^{-1}$ and $\mu_{\delta}=-8.87 \pm 1.77$ mas yr $^{-1}$ (Perryman et al. 1997). These values agree with the weighted average obtained by us at the $\pm 1-\sigma$ level.

Our results confirm that the source VLA 3 is a member of the NGC 1333 molecular cloud since this source shows the same proper motion that the other members of the cloud. Although this conclusion alone does not resolve the problem of the identification of the powering source of the HH 7-11 system, our results discard the possibility that VLA 3 is a foreground or background source not related to the NGC 1333 region. Therefore, the proximity of VLA 3 to the base of the $\mathrm{HH}$ 7-11 system is physically real, and not only a projection effect.

The determination of the proper motions of embedded young stars in NGC 1333 is useful to reanalyze the proper motions of water masers reported by Hirota et al. (2008). These authors report VLBI observations of two maser features associated with VLA 4A (SVS 13). They found absolute proper motions of the order of 15-20 mas $\mathrm{yr}^{-1}$ in a direction (P.A. $\simeq 114^{\circ}-133^{\circ}$ ) roughly aligned with the jets and outflows in the region. However, as noted by Hirota et al. (2008), these proper motions contain a significant contribution due to the relative motion of the NGC 1333 region with respect to the Sun. In order to correct for the contribution due to the relative motion of the NGC 1333 region with respect to the Sun, Hirota et al. (2008) subtracted the theoretical proper motion of the region due to the Solar motion relative to the LSR (neglecting the contribution of Galactic rotation, that they show is small). This correction results in proper motions of the order of 7-14 mas $\mathrm{yr}^{-1}$, and a significant change in the direction. The proper motions corrected in this way are not parallel to the $\mathrm{HH}$ 7-11 jet and $\mathrm{CO}$ outflow. However, as we have discussed above, there is still a significant contribution due to the peculiar proper motion of the NGC 1333 region that must be taken into account for a proper interpretation of the results.

Our results show that the mean proper motion of the NGC 1333 region $\left(\mu_{\alpha} \cos \delta=9 \pm 1\right.$ mas $\mathrm{yr}^{-1}$ and $\mu_{\delta}=$

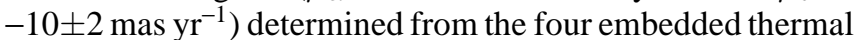
sources is comparable to the absolute proper motions found by Hirota et al. (2008) for the two water maser features as-

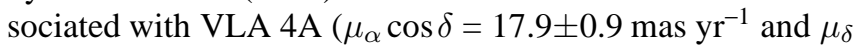

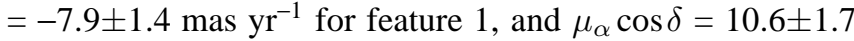

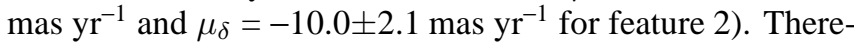
fore, this correction appears to be very important to obtain the true proper motions relative to the region. The close coincidence for feature 2 suggests that this maser is almost stationary with respect to the cloud and that is not tracing significant peculiar proper motions. This conclusion is supported by the fact that the LSR velocity of the masers $\left(7-8 \mathrm{~km} \mathrm{~s}^{-1}\right)$ nearly coincides with the LSR velocity of the molecular cloud, as already noted by Rodríguez et al. (2002). After correction for the proper motion of the NGC 1333 region determined by us, feature 1 has residual proper motions of $\mu_{\alpha} \cos \delta=$

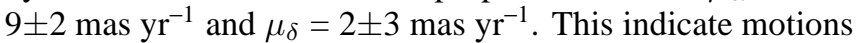
to the east $\left(\right.$ P.A. $\simeq 90^{\circ}$ ) with a velocity of $\sim 10 \mathrm{~km} \mathrm{~s}^{-1}$, that align poorly with the chain of bright $\mathrm{HH} 7-11$ objects and the molecular outflow. In any case, these small residual proper motions align much better with the faint chain of $\mathrm{HH}$ objects (a, b, c and d) found by Davis et al. (1995), that aligns in the west-east direction (see Fig. 2 of Rodríguez et al. 1997 where the positions of the sources discussed in this paper and the $\mathrm{HH}$ objects are also shown).

We note, from the analysis discussed above, that we obtained proper motions of the maser features relative to the NGC1333 cloud. An unambiguous measurement of the proper motions relative to VLA 4A would require to subtract the individual proper motion of this source instead of the average motion of the NGC 1333 region. The nominal proper motion velocity we obtained for this source is about one half the average value of the region. Taken at face value, it could suggest that the VLA 4A source has a significant velocity relative to the ambient cloud. However, the weakness of the source does not allow a reliable determination of its individual proper motion with the currently available data. Additional, high sensitivity data would be required to investigate possible motions of the VLA 4A source relative to the NGC 1333 cloud in order to accurately determine the motions of the water maser features relative to VLA 4A.

\section{CONCLUSIONS}

We have presented the results of multi-epoch high angular resolution VLA observations at $3.6 \mathrm{~cm}$ of the NGC 1333 star forming region. From these observations we have measured the absolute positions at different epochs of four thermal sources (VLA 2, VLA 3, VLA 4A and VLA 4B) tracing YSOs embedded in this region. All these sources show an absolute proper motion to the SE, which we interpret as a proper motion of the NGC 1333 region as a whole. From our data we

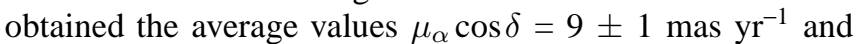

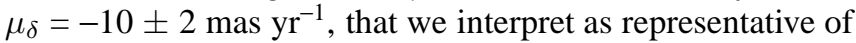
the proper motion of the molecular cloud associated with the NGC 1333 region as a whole.

Our results allow us to correct the proper motion measurements of water maser spots associated with VLA 4A (SVS 13) obtained by Hirota et al. (2008) in order to obtain the proper motions relative to the NGC 1333 cloud. We conclude that feature 2 of Hirota et al. (2008) is practically stationary with respect to the cloud, and that the small residual proper motions of feature 1 indicate motions to the east, suggesting that none of these features is associated with the HH 7-11 system or the molecular outflow.

Our data also suggest that VLA 3 is a member of the NGC 1333 molecular cloud, since this source shows a proper motion similar to that of the others sources embedded in the region. Since the position of VLA 3 appears close to the base of the $\mathrm{HH} 7-11$ system, we conclude that this proximity is physically real, and not a projection effect.

Acknowledgements. C.C.-G. acknowledges support from MEC (Spain) FPU fellowship. G.A., C.C.-G., M.O., and J.M.T. acknowledge support from MEC (Spain) grants AYA 2005-08523-C03 and AYA 2008-06189-C03 (including FEDER funds), and from Junta de Andalucía (Spain). L.F.R. acknowledges the support of DGAPA, UNAM, and of CONACyT (México). We thank Tomoya Hirota for useful comments on this paper. 


\section{REFERENCES}

Anglada, G., Rodríguez, L. F., \& Torrelles, J. M. 2000, ApJ, 542, L123

Anglada, G., Rodríguez, L. F., Osorio, M., Torrelles, J. M., Estalella, R., Beltrán, M. T., \& Ho, P. T. P. 2004, ApJ, 605, L140

Bachiller, R., Gueth, F., Guilloteau, S., Tafalla, M., Dutrey, A. 2000, A\&A, $362, \mathrm{~L} 33$

Cernis, K. 1990, Ap\&SS, 166, 315

Curiel, S., Girart, J. M., Rodríguez, L. F., \& Cantó, J. 2003, ApJ, 582, L109

Davis, C. J., Mundt, R., Eislöfel, J., \& Ray, T. 1995, AJ, 110, 766

Dehnen, W., Binney, J. J. 1998, MNRAS, 298, 387

Gómez, L., Rodríguez, L.F., Loinard, L., Poveda, A., Lizano, S., \& Allen, C. 2005, ApJ, 635, 1166

Herbig, G. H., Jones, B. F. 1983, AJ, 88, 1040

Hirota, T., Bushimata, T., Choi, Y. K., Honma, M., Imai, H., Iwadate, K., Jike,

T., Kameya, O., Kamohara, R., Kan-ya, Y., Kawaguchi, N., Kijima, M., Kobayashi, H., Kuji, S., Kurayama, T., Manabe, S., Miyaji, T., Nagayama, T., Nakagawa, A., Oh, C. S., Omodaka, T., Oyama, T., Sakai, S., Sasao, T., Sato, K., Shibata, K. M., Tamura, Y., Yamashita, K. 2008, PASJ, 60, 37 Loinard, L., Rodríguez, L. F., D’Alessio, P., Wilner, D. J., \& Ho, P. T. P. 2002 , ApJ, 581, L109
Loinard, L., Rodríguez, L. F., \& Rodríguez, M. I. 2003, ApJ, 587, L47

Loinard, L., Torres, R. M., Mioduszewski, A. J., Rodríguez, L. F., GonzálezLópezlira, R. A., Lachaume, R., Vázquez, V., \& González, E. 2007, ApJ, 671,546

Looney, L. W., Mundy, L. G.,\& Welch, W. J. 2000, ApJ, 529, 477

Perryman, M .A. C., Lindegren, L., Kovalevsky, J., Hoeg, E., Bastian, U., Bernacca, P. L., Crézé, M., Donati, F., Grenon, M., van Leeuwen, F., van der Marel, H., Mignard, F., Murray, C. A., Le Poole, R. S., Schrijver, H., Turon, C., Arenou, F., Froeschlé, M., Petersen, C. 1997, A\&A, 323, 49

Rodríguez, L.F., Escalante, V., Lizano, S., Cantó, J., Mirabel, I.F. 1990, ApJ, 365,261

Rodríguez, L. F., Anglada, G., \& Curiel, S. 1997, ApJ, 480, L125

Rodríguez, L. F., Anglada, G., Torrelles, J. M., Mendoza-Torres, J. E., Haschick, A. D., \& Ho, P. T. P. 2002, A\&A, 389, 572

Rodríguez, L. F., Curiel, S., Cantó, J., Loinard, L., Raga, A. C., \& Torrelles, J. M. 2003, ApJ, 583, 330

Rodríguez, L. F., Poveda, A., Lizano, S., \& Allen, C. 2005, ApJ, 627, L65

Strom, S. E., Vrba, F. J.,\& Strom, K. M. 1976, AJ, 81, 314 